\title{
Beam Spin Asymmetry in Semi-Inclusive Electroproduction of Hadron Pairs
}

M. Mirazita $\odot,{ }^{19}$ H. Avakian, ${ }^{42}$ A. Courtoy, ${ }^{15}$ S. Pisano, ${ }^{5}$ S. Adhikari, ${ }^{13}$ M. J. Amaryan, ${ }^{35}$ Giovanni Angelini,${ }^{16}$ H. Atac, ${ }^{41}$ N. A. Baltzell, ${ }^{42}$ L. Barion, ${ }^{18}$ M. Battaglieri, ${ }^{42,20}$ I. Bedlinskiy, ${ }^{31}$ Fatiha Benmokhtar, ${ }^{10}$ A. Bianconi, ${ }^{45,23}$ A. S. Biselli, ${ }^{11,3}$ F. Bossù, ${ }^{6}$ S. Boiarinov, ${ }^{42}$ W. J. Briscoe, ${ }^{16}$ W. K. Brooks, ${ }^{43,42}$ D. Bulumulla, ${ }^{35}$ V. D. Burkert, ${ }^{42}$ D. S. Carman, ${ }^{42}$ J. C. Carvajal, ${ }^{13}$ A. Celentano, ${ }^{20}$ P. Chatagnon, ${ }^{24}$ T. Chetry, ${ }^{30}$ G. Ciullo, ${ }^{18,12}$ B. Clary, ${ }^{8}$ P. L. Cole, ${ }^{28,42}$ M. Contalbrigo,${ }^{18}$ V. Crede, ${ }^{14}$ A. D'Angelo, ${ }^{21,38}$ N. Dashyan, ${ }^{51}$ R. De Vita, ${ }^{20}$ M. Defurne, ${ }^{6}$ A. Deur, ${ }^{42}$ S. Diehl,${ }^{8}$ C. Dilks, ${ }^{9}$ C. Djalali, ${ }^{34,40}$ R. Dupre, ${ }^{24}$ H. Egiyan, ${ }^{42}$ M. Ehrhart, ${ }^{1}$ A. El Alaoui ${ }^{43}$ L. El Fassi, ${ }^{30}$ P. Eugenio, ${ }^{14}$ S. Fegan, ${ }^{47}$ R. Fersch,${ }^{7,50}$ A. Filippi,${ }^{22}$ T. A. Forest, ${ }^{17}$ Y. Ghandilyan, ${ }^{51}$ G. Gavalian, ${ }^{42,32}$ G. P. Gilfoyle, ${ }^{37}$ K. L. Giovanetti, ${ }^{26}$ F. X. Girod, ${ }^{42}$ D. I. Glazier, ${ }^{46}$ E. Golovatch, ${ }^{39}$ R. W. Gothe ${ }^{40}$ K. A. Griffioen, ${ }^{50}$ M. Guidal,${ }^{24}$ L. Guo, ${ }^{13,42}$ K. Hafidi, ${ }^{1}$ H. Hakobyan, ${ }^{43,51}$ M. Hattawy, ${ }^{35}$ T. B. Hayward, ${ }^{50}$ D. Heddle, ${ }^{7,42}$ K. Hicks, ${ }^{34}$ A. Hobart, ${ }^{24}$ M. Holtrop, ${ }^{32}$ Q. Huang, ${ }^{6}$ Y. Ilieva, ${ }^{40,16}$ D. G. Ireland, ${ }^{46}$ B. S. Ishkhanov, ${ }^{39}$ E. L. Isupov, ${ }^{39}$ D. Jenkins, ${ }^{48}$ H. S. Jo, ${ }^{27,24}$ K. Joo, ${ }^{8}$ D. Keller, ${ }^{49}$ A. Khanal, ${ }^{13}$ M. Khandaker $,{ }^{33,},{ }^{*}$ C. W. Kim,${ }^{16}$ W. Kim,${ }^{27}$ F. J. Klein ${ }^{4}$ V. Kubarovsky, ${ }^{42,36}$ S. E. Kuhn, ${ }^{35}$ L. Lanza, ${ }^{21}$ M. Leali, ${ }^{45,23}$ P. Lenisa, ${ }^{18,12}$ K. Livingston, ${ }^{46}$ I. J. D. MacGregor, ${ }^{46}$ D. Marchand,${ }^{24}$ N. Markov, ${ }^{42,8}$ L. Marsicano,${ }^{20}$ V. Mascagna, ${ }^{44,23, \dagger}$ B. McKinnon, ${ }^{46}$ R. G. Milner, ${ }^{29}$ T. Mineeva, ${ }^{43}$ V. Mokeev, ${ }^{42,39}$ C. Mullen, ${ }^{20}$ C. Munoz Camacho,${ }^{24}$ K. Neupane, ${ }^{40}$ G. Niculescu, ${ }^{26,34}$ T. O'Connell, ${ }^{8}$ M. Osipenko, ${ }^{20}$ M. Paolone, ${ }^{41}$ L. L. Pappalardo, ${ }^{18,12}$ R. Paremuzyan, ${ }^{42}$ K. Park, ${ }^{27, \ddagger}$ E. Pasyuk ${ }^{42}$ W. Phelps ${ }^{7}$ D. Pocanic,${ }^{49}$ O. Pogorelko, ${ }^{31}$ J. Poudel,${ }^{35}$ Y. Prok,${ }^{35,49}$ B. A. Raue, ${ }^{13,42}$ M. Ripani ${ }^{20}$ J. Ritman, ${ }^{25}$ A. Rizzo,${ }^{21,38}$ P. Rossi,${ }^{42,19}$ F. Sabatié, ${ }^{6}$ C. Salgado, ${ }^{33}$ A. Schmidt, ${ }^{16}$ R. A. Schumacher, ${ }^{3}$ Y. G. Sharabian, ${ }^{42}$ U. Shrestha, ${ }^{34}$ O. Soto, ${ }^{19}$ N. Sparveris,${ }^{41}$ S. Stepanyan, ${ }^{42}$ I. I. Strakovsky, ${ }^{16}$ S. Strauch, ${ }^{40,16}$ N. Tyler, ${ }^{40}$ M. Ungaro, ${ }^{42,36}$ L. Venturelli, ${ }^{45,23}$ H. Voskanyan, ${ }^{51}$ A. Vossen, ${ }^{9}$ E. Voutier, ${ }^{24}$ D. Watts, ${ }^{47}$ K. Wei, ${ }^{8}$ X. Wei, ${ }^{42}$ M. H. Wood, ${ }^{2,40}$ B. Yale, ${ }^{50}$ N. Zachariou, ${ }^{47}$ J. Zhang, ${ }^{49}$ and Z. W. Zhao ${ }^{9}$

\section{(CLAS Collaboration)}

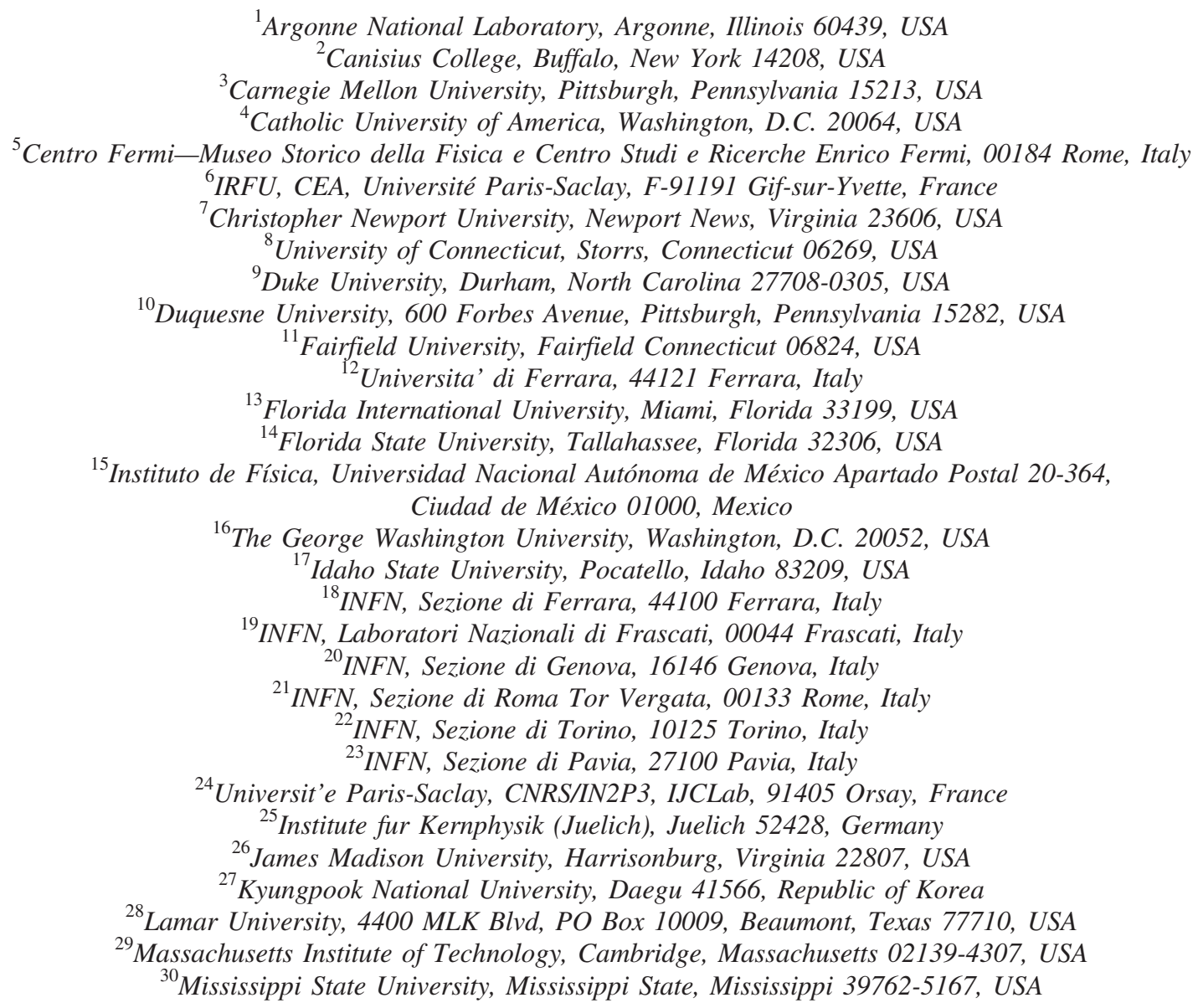




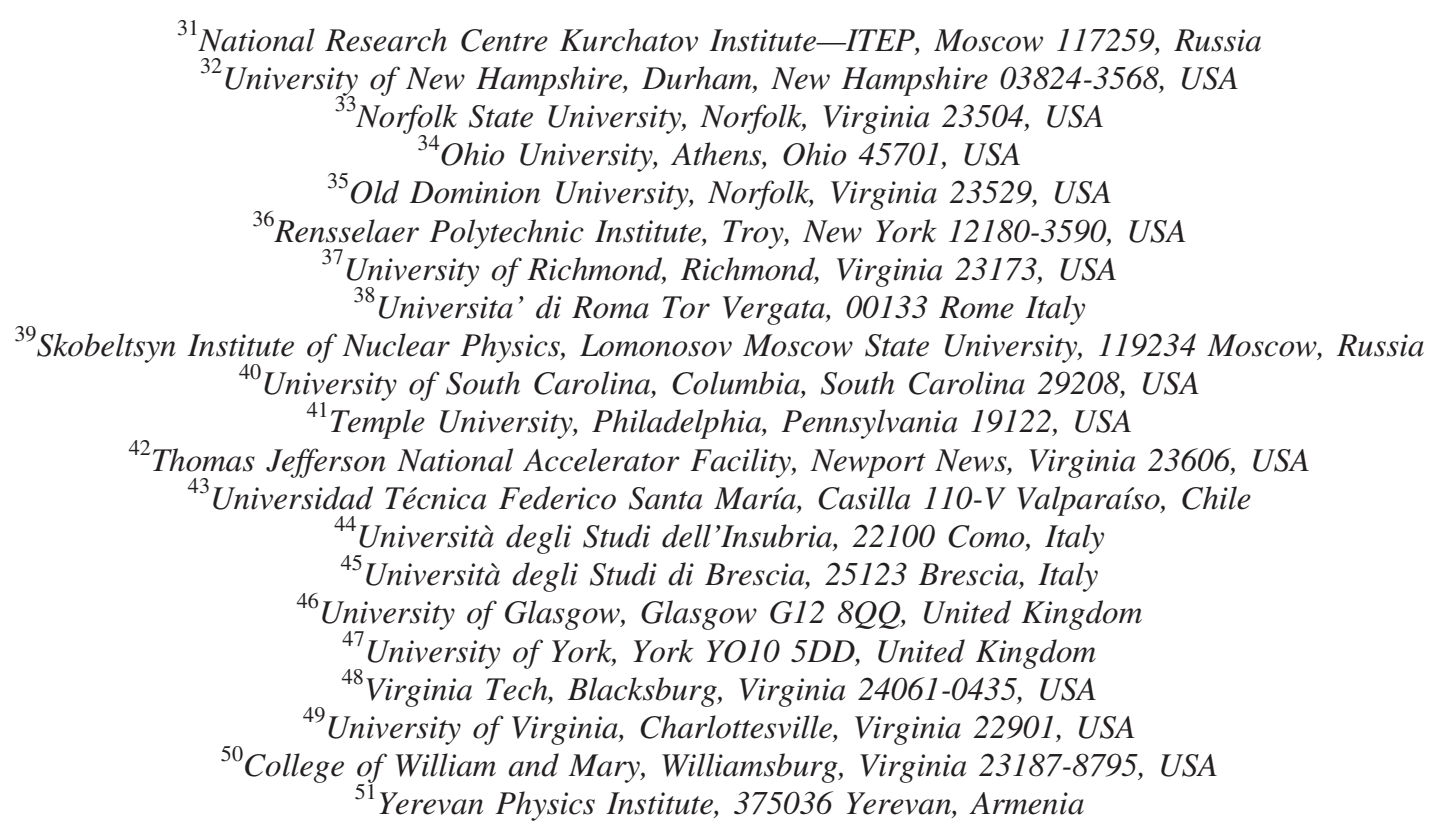

(Received 26 October 2020; revised 23 December 2020; accepted 12 January 2021; published 12 February 2021)

A first measurement of the longitudinal beam spin asymmetry $A_{L U}$ in the semi-inclusive electroproduction of pairs of charged pions is reported. $A_{L U}$ is a higher-twist observable and offers the cleanest access to the nucleon twist-3 parton distribution function $e(x)$. Data have been collected in the Hall-B at Jefferson Lab by impinging a 5.498-GeV electron beam on a liquid-hydrogen target, and reconstructing the scattered electron and the pion pair with the CLAS detector. One-dimensional projections of the $A_{L U}^{\sin \phi_{R}}$ moments are extracted for the kinematic variables of interest in the valence quark region. The understanding of dihadron production is essential for the interpretation of observables in single-hadron production in semi-inclusive DIS, and pioneering measurements of single-spin asymmetries in dihadron production open a new avenue in studies of QCD dynamics.

DOI: 10.1103/PhysRevLett.126.062002

The correlations between quarks and gluons occurring inside the nucleon play an essential role in QCD dynamics. Asymmetries from semi-inclusive deep inelastic scattering (SIDIS), where a highly virtual photon interacts with a hadronic target and at least one hadron is detected in the final state, have appeared to be effective tools to access quark distributions and fragmentation information. Studies of hadron pairs in SIDIS open qualitatively new possibilities to study QCD dynamics, providing access to correlations not accessible with single-hadron SIDIS. The interpretation of dihadron production in SIDIS, as well as interpretation of single-hadron production, are intimately related to contributions to those samples from correlated dihadrons in general, and vector mesons, in particular.

Published by the American Physical Society under the terms of the Creative Commons Attribution 4.0 International license. Further distribution of this work must maintain attribution to the author(s) and the published article's title, journal citation, and DOI. Funded by SCOAP.
At the energy of fixed-target facilities, contributions of order $\mathcal{O}(M / Q)$, with $M$ the target mass and $Q^{2}$ the photon virtuality, become sizable and, therefore, relevant. Such contributions are labeled twist-3 effects, and can encode quark-gluon correlations.

In the collinear framework-in which we shall work in this Letter-six parton distribution functions (PDFs) describe the nucleon up to the twist-3 level. The three leading-twist functions are the unpolarized $f_{1}(x)$, the helicity $g_{1}(x)$, and the tranversity $h_{1}(x)$ distributions, where $x$ is the Bjorken scaling variable. The twist-3 PDFs are $e(x), h_{L}(x)$, and $g_{T}(x)$, which describe quark-quark and quark-gluon correlations, and as such have no probabilistic interpretation.

Higher-twist PDFs offer fascinating doorways to study the nucleon beyond its valence structure. An essential role is played by the chiral-odd PDF $e(x)$, that is related to the nucleon scalar charge-poorly determined through phenomenology - and a fortiori to the pion-nucleon sigma term [1]. In addition, it encodes information on the quark mass, as well as genuine quark-gluon correlations [2]. 
The latter has also been related to an average transverse force acting on a transversely polarized quark in an unpolarized target after the interaction with the virtual photon [3]. It has recently been suggested that this effect could be related to the $C P$-violating sigma terms [4]. Consequently, the precise measurement of the PDF $e(x)$ could play an important role in searches for beyond-theStandard-Model fundamental scalar interactions [5], in the same way the tensor charge does [6].

Measurements of twist-3 observables are available from HERMES [7], CLAS [8-10], and COMPASS [11] for single-pion SIDIS, for which the PDF $e(x)$ is accessible only when the transverse momentum of the final hadron is not integrated out. A first extraction of the-transverse momentum dependent- $e(x)$ PDF has been pursued from the CLAS data [12]. Two-hadron SIDIS measurements provide a method to access twist-3 observables in the collinear framework.

In this Letter, we present the first measurement of the beam-spin asymmetry (BSA) in the SIDIS electroproduction of two charged pions using the CLAS data. The BSA is defined as the ratio between the difference and the sum of the cross sections corresponding to the two beamhelicity states and can be expressed in terms of harmonics in the azimuthal angle $\phi_{R}$, defined below. The $\sin \phi_{R}$ moment of the BSA can be written as

$$
A_{L U}^{\sin \phi_{R}}=\sqrt{2 \epsilon(1-\epsilon)} \frac{F_{L U}^{\sin \phi_{R}}}{F_{U U, T}+\epsilon F_{U U, L}},
$$

with $\epsilon$ the ratio of longitudinal to transverse photon flux, and the structure functions (SFs) [13]

$$
\begin{aligned}
& F_{L U}^{\sin \phi_{R}}=-\sum_{q} e_{q}^{2} x \frac{|\boldsymbol{R}| \sin \theta}{Q} \\
& {\left[\frac{M}{m_{\pi^{+} \pi^{-}}} x e^{q}(x) H_{1}^{\measuredangle q}\left(z, \cos \theta, m_{\pi^{+} \pi^{-}}\right)\right.} \\
&\left.+\frac{1}{z} f_{1}^{q}(x) \tilde{G}^{\measuredangle q}\left(z, \cos \theta, m_{\pi^{+} \pi^{-}}\right)\right], \\
& F_{U U, T}= \sum_{q} e_{q}^{2} x f_{1}^{q}(x) D_{1}^{q}\left(z, \cos \theta, m_{\pi^{+} \pi^{-}}\right), \\
& F_{U U, L}=0,
\end{aligned}
$$

contain a sum over the quark flavors $q$ with charge $e_{q}$, with $M$ the target mass and $m_{\pi^{+} \pi^{-}}$the invariant mass of the pion pair. The angles are calculated in the center of mass frame of the virtual photon-proton system. We define the sum of the two pion momenta $\mathbf{P}_{h}=\mathbf{P}_{\pi^{+}}+\mathbf{P}_{\pi^{-}}$as well as their half-difference $\mathbf{R}=\left(\mathbf{P}_{\pi^{+}}-\mathbf{P}_{\pi^{-}}\right) / 2$. The azimuthal angle $\phi_{R}$ is defined through the plane formed by the spatial component of $\mathbf{R}$ orthogonal to $\mathbf{P}_{h}$

$$
\boldsymbol{R}_{T}=\boldsymbol{R}-\left(\boldsymbol{R} \cdot \hat{\boldsymbol{P}}_{h}\right) \hat{\boldsymbol{P}}_{h},
$$

and the virtual photon direction, i.e.,

$$
\phi_{R}=\frac{(\boldsymbol{q} \times \boldsymbol{k}) \cdot \boldsymbol{R}_{T}}{\left|(\boldsymbol{q} \times \boldsymbol{k}) \cdot \boldsymbol{R}_{T}\right|} \arccos \left(\hat{n}_{\mathrm{lept}} \cdot \hat{n}_{\mathrm{had}}\right) .
$$

The azimuthal angle $\phi_{h}$ is defined through the plane formed by $\mathbf{P}_{h}$ and the direction of the virtual photon and will be important for the study of acceptance effects. Both angles, $\phi_{h}$ and $\phi_{R}$, correspond to the definitions given in Ref. [14].

Each SF has two subscripts indicating the polarization of the beam and target, respectively, and can be written in terms of simple products of a PDF and a dihadron fragmentation function (FF). The SFs depend on the Bjorken variable $x$ (through the PDF) and on the fraction of the virtual photon energy carried by the two hadrons $z=z_{\pi^{+}}+z_{\pi^{-}}$, on the pion pair invariant mass $m_{\pi^{+} \pi^{-}}$, and on $\cos \theta$ (through the FF). Here, $\theta$ is the angle between the direction of one of the final state hadrons in the pair center of mass frame and the direction of their center of mass in the photon-target rest frame. The PDFs and FFs depend also on $Q^{2}$, through the kinematical suppression of the twist-3 contributions, as well as the QCD evolution equations.

The unpolarized cross section is proportional to the product of the unpolarized PDF $f_{1}(x)$ and the unpolarized dihadron FF $D_{1}$. We neglect here the structure function $F_{U U}^{\cos \phi_{R}}$, which is considered in the systematic uncertainty evaluation only. The two terms appearing in the polarized structure function $F_{L U}^{\sin \phi_{R}}$ are both the product of a leadingtwist function and a twist-3 one, which could equally contribute. In Eq. (2), $e(x)$ appears coupled to the chiralodd interference fragmentation function (IFF) $H_{1}^{<}$[13].

In the dihadron framework $[15,16]$, the hadron pair is assumed to be mainly produced in a relative $s$ - or $p$-wave channel. Therefore, it is convenient to expand the $\cos \theta$ dependence of the IFF in a partial wave series that can be truncated to the first-order terms. This allows, for example, to replace the IFF $H_{1}^{\triangleleft}\left(z, \cos \theta, m_{\pi^{+} \pi^{-}}\right)$in Eq. (2) with the leading term of the partial wave expansion $H_{1, s p}^{女}\left(z, m_{\pi^{+} \pi^{-}}\right)$, which has been extracted [17-19] from Belle data [20].

The analysis we present in this Letter is based on the data collected in Hall B of Jefferson Laboratory in 2003 by impinging a $5.498 \mathrm{GeV}$ longitudinally polarized electron beam on a 5-cm long unpolarized liquid-hydrogen target. The CEBAF Large Acceptance Spectrometer (CLAS) [21] was used to detect the scattered electron and two oppositely charged pions of the reaction $e p \rightarrow e \pi^{+} \pi^{-} X$. The final dihadron sample is defined through specific DIS selection criteria. To be in the scaling regime, the virtuality of the exchanged photon is required to be $Q^{2}>1 \mathrm{GeV}^{2}$ and, in order to avoid the resonance region, we impose $W>2 \mathrm{GeV}$. The cut $y<0.85$ is then applied to suppress radiative events. Pions coming from the 
current-fragmentation region are selected by applying on each pion the cut $x_{F}>0$, where the Feynman- $x$ variable is defined as $x_{F}=2 p_{\|} / W$, with $p_{\|}$the pion pair fourmomentum component parallel to the virtual-photon direction. Exclusive events are removed through a cut on the missing mass $m_{e \pi^{+} \pi^{-} X}>1.05 \mathrm{GeV}$. Spurious contaminations from exclusive baryonic resonance production (for example $\Delta^{++} \pi^{-} \rightarrow p \pi^{+} \pi^{-}$) have been studied through Monte Carlo simulations and are further suppressed at the few percent level by cutting on the energy fraction of the pions, namely, $z_{\pi^{+}}>0.28$ and $z_{\pi^{-}}>0.25$.

Experimentally, the BSA is defined as

$$
A_{L U}=\frac{1}{P_{B}} \frac{\left(N_{+}-N_{-}\right)}{\left(N_{+}+N_{-}\right)},
$$

where $N_{+(-)}$are the number of counts corresponding to each beam-helicity state, and $P_{B}=0.75 \pm 0.02$ is the average beam polarization over the entire data taking period. The BSA has been computed in one-dimensional projections as a function of $x, z, m_{\pi^{+} \pi^{-}}$, and $Q^{2}$ and integrating over all the other kinematic variables.

From the theoretical point of view, the only surviving azimuthal modulation of the BSA is the $\sin \phi_{R}$ moment of Eq. (1). However, kinematic correlations due to the limited phase space available in real data and nonuniform detector acceptance might lead to incomplete cancellation of modulations involving transverse momentum dependent functions [16]. A detailed Monte Carlo simulation study using an event generator based on PYTHIA and JETSET [22] and a full GEANT4 [23] simulation of CLAS demonstrated that a reliable extraction of the $A_{L U}^{\sin \phi_{R}}$ moment can be achieved by binning the data in a $6 \times 6$ matrix in the two angles $\phi_{R}$ and $\Delta \phi=\phi_{h}-\phi_{R}$, and performing a $2 \mathrm{D}$ fit with the function

$$
\begin{aligned}
A_{L U}= & A_{L U}^{\sin \phi_{R}} \sin \phi_{R} \\
& +A_{L U}^{\sin \left(\phi_{h}-\phi_{R}\right)} \sin \left(\phi_{h}-\phi_{R}\right)+A_{L U}^{\sin \phi_{h}} \sin \phi_{h},
\end{aligned}
$$

representing the relevant modulations from the cross section.

An example of the $2 \mathrm{D}$ fit in the bin $x=0.2 \div 0.3$ is shown in Fig. 1. Each panel represents one $\Delta \phi$ bin, the points show the $\phi_{R}$ dependence of the measured BSA. The curve is the result of the 2D fit. The fit has been performed considering the total (statistical and systematic) point-topoint uncertainty shown by the error bars. The systematic uncertainty, amounting to $30 \%$, is due to the truncation in the partial wave expansion of the dihadron FFs. It has been estimated by taking into account the average values of the $\theta$ harmonics of the series from the experimental data and conservatively assuming that the FFs associated to higher harmonics are of the same order of magnitude as the leading one.
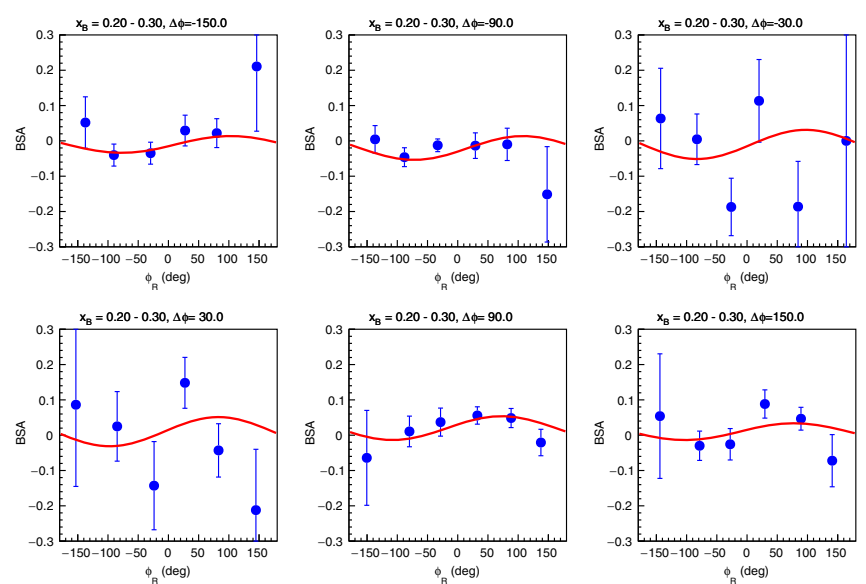

FIG. 1. BSA as a function of $\phi_{R}$ in the $6 \Delta \phi$ bins from $-180^{\circ}$ to $180^{\circ}$ for the bin $x=0.2 \div 0.3$. The full circles represent the experimental measurement with the vertical bar indicating the total uncertainty, while the curves represent the result of the fit with Eq. (8).

The $A_{L U}^{\sin \phi_{R}}$ fitted moments are shown in Fig. 2 as a function of $x$ and $Q^{2}$. The projections for $z$ and $m_{\pi^{+} \pi^{-}}$are given in Fig. 3. The solid circles correspond to data points. The error bars show the statistical uncertainties from the fits. At the bottom of each plot, the gray band represents the total systematic uncertainty, which includes (summed up quadratically): a $3 \%$ contribution due to the electron beam polarization; a $3 \%$ contribution due to the radiative corrections; the residual contamination from baryon resonance decays, estimated from Monte Carlo studies to be between $2 \%$ and $9 \%$ depending on the kinematics.

The kinematic bins in the figures have been chosen so that they have approximately the same statistics, except for $m_{\pi^{+} \pi^{-}}$, where the second bin covers the $\rho$ mass region, while the first (third) bin covers the mass region before (after) the $\rho$ mass. The average $Q^{2}$ of the data is $1.77 \mathrm{GeV}^{2}$. For the $x$ dependence of the BSA, the invariant mass values range from the threshold to $\sim 1.7 \mathrm{GeV}$ and $0.53<z<0.95$.

In this Letter we report the pioneering observation of a twist-3 observable in dihadron SIDIS; hence, no previous
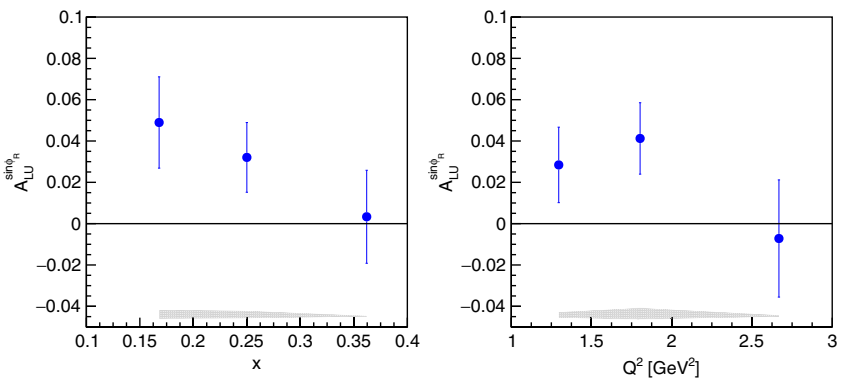

FIG. 2. Dependence on $x$ (left) and $Q^{2}$ (right) of the $\sin \phi_{R}$ moment $A_{L U}^{\sin \phi_{R}}$ of the BSA. The error bars and the gray band represents the statistical and systematic uncertainties. 

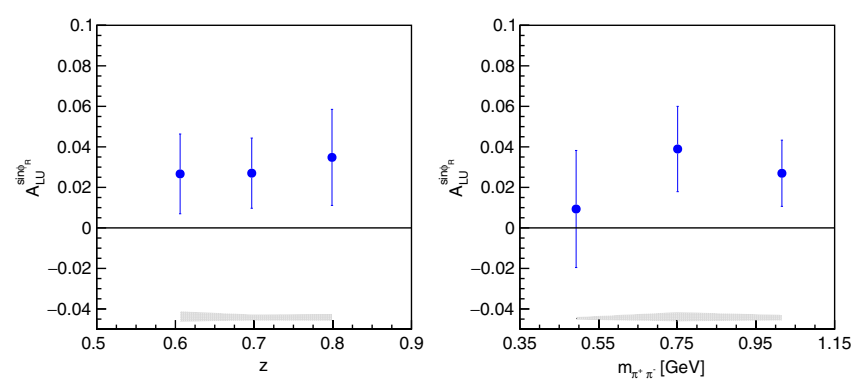

FIG. 3. Dependence on $z$ (left) and $m_{\pi \pi}$ (right) of the $\sin \phi_{R}$ moment $A_{L U}^{\sin \phi_{R}}$ of the BSA. The error bars and the gray band represents the statistical and systematic uncertainties.

measurements are available for comparison. The $x$ projection of the $\sin \phi_{R}$ moment reflects the behavior of the PDFs. In Fig. 4 this projection of $A_{L U}^{\sin \phi_{R}}$ is compared to models for the twist-3 PDF $e(x)$-neglecting the second term on the right-hand side of Eq. (2). The curves corresponding to the different models for $e(x)$ are produced by combining to the extracted dihadron FFs [19]. The gray-dotted band corresponds to the LFCQM of Ref. [24] together with MSTW08LO unpolarized PDF [25], the green-dashed band corresponds to the asymmetry with both PDFs evaluated in the spectator model [26], while the red-full band corresponds to the MIT bag model [27] for both $f_{1}(x)$ and $e(x)$. Other calculations, not shown in the figure, have been proposed [28-30]. The models are consistent among themselves; they are in agreement with the experimental data within about 1 standard deviation, although the data seem to indicate a steeper decrease at high $x$ than the models. The behavior of the three points could be examined in a thorough pointby-point extraction of $e(x)$, as has been sketched in Ref. [31] using preliminary results from the same CLAS data we present in this Letter.

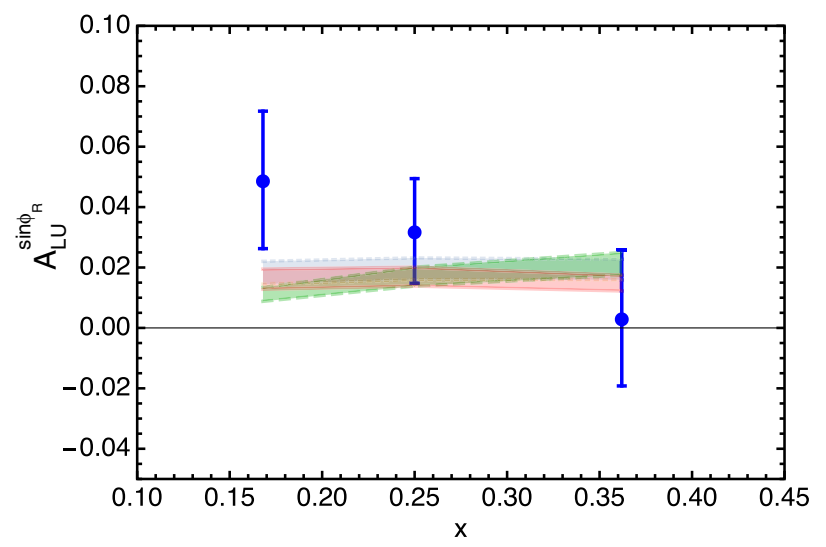

FIG. 4. Dependence on $x$ of the $\sin \phi_{R}$ moment $A_{L U}^{\sin \phi_{R}}$ of the beam-spin asymmetry compared to model calculations: the spectator model [26] in the green-dashed band, the bag model [27] in the red-full band, and the gray-dotted band corresponds to the LFCQM [24], all use the dihadron FFs [19].
In summary, for the first time a beam-spin asymmetry for the semi-inclusive electroproduction of charged pion pairs has been measured. The one-dimensional projections of the moment $A_{L U}^{\sin \phi_{R}}$ in $x, z, m_{\pi^{+} \pi^{-}}$and $Q^{2}$ have been extracted. This measurement constitutes a pioneering study that has accounted for systematic sources of uncertainties that were shown to have little impact on the present results but that would be crucial for ongoing studies of dihadron observables using the CLAS12 [32] detector with proton and deuteron targets [33], at an order of magnitude higher luminosity, which will provide further information on the twist-3 nucleon structure in the mid- $x$ region.

The invariant mass distributions of dihadrons from different SIDIS and $e^{+} e^{-}$experiments indicate that a very significant fraction of inclusive pions are coming from correlated dihadrons. The observables for pions from decays of vector mesons have peculiar spin and momentum dependences and may require different radiative corrections, modeling, and interpretation of observables sensitive to the transverse momentum of quarks [34]. As such, dihadron measurements establish the bases for future experimental and phenomenological studies.

We thank the staff of the Accelerator and Physics Divisions for making the experiment possible. Special thanks to A. Bacchetta, B. Pasquini, M. Polyakov, M. Radici, P. Schweitzer, and M. Wakamatsu for useful discussions. This work was supported in part by the U.S. Department of Energy (No. DE-FG02-96ER40950) and National Science Foundation, the French Centre National de la Recherche Scientifique and Commissariat à l'Energie Atomique, the French-American Cultural Exchange (FACE), the Italian Istituto Nazionale di Fisica Nucleare, the Chilean Comisión Nacional de Investigación Científica y Tecnológica (CONICYT), the Mexican Consejo Nacional de Ciencias y Tecnología (CONACyT), the National Research Foundation of Korea, and the UK Science and Technology Facilities Council (STFC). A. C. is supported by DGAPA-PAPIIT IA101720 and CONACyT Ciencia de Frontera No. 51244 (FORDECYTPRONACES). The Jefferson Science Associates (JSA) operates the Thomas Jefferson National Accelerator Facility for the United States Department of Energy under Contract No. DE-AC05-06OR23177.

*Current address: Idaho State University, Pocatello, Idaho 83209, USA.

Current address: Università degli Studi di Brescia, 25123 Brescia, Italy.

Current address: Thomas Jefferson National Accelerator Facility, Newport News, Virginia 23606, USA.

[1] R. L. Jaffe and X. D. Ji, Nucl. Phys. B375, 527 (1992).

[2] A. Efremov and P. Schweitzer, J. High Energy Phys. 08 (2003) 006.

[3] M. Burkardt, Phys. Rev. D 88, 114502 (2013). 
[4] C. Y. Seng, Phys. Rev. Lett. 122, 072001 (2019).

[5] J. R. Ellis, K. A. Olive, and C. Savage, Phys. Rev. D 77, 065026 (2008); T. Bhattacharya, V. Cirigliano, S. D. Cohen, A. Filipuzzi, M. Gonzalez-Alonso, M. L. Graesser, R. Gupta, and H. W. Lin, Phys. Rev. D 85, 054512 (2012); M. Cirelli, E. Del Nobile, and P. Panci, J. Cosmol. Astropart. Phys. 10 (2013) 019.

[6] A. Courtoy, S. Baessler, M. Gonzalez-Alonso, and S. Liuti, Phys. Rev. Lett. 115, 162001 (2015); T. Liu, Z. Zhao, and H. Gao, Phys. Rev. D 97, 074018 (2018).

[7] A. Airapetian et al. (HERMES Collaboration), Phys. Lett. B 648, 164 (2007).

[8] W. Gohn et al. (CLAS Collaboration), Phys. Rev. D 89, 072011 (2014).

[9] H. Avakian et al. (CLAS Collaboration), Phys. Rev. D 69, 112004 (2004).

[10] M. Aghasyan et al., Phys. Lett. B 704, 397 (2011).

[11] A. Moretti (COMPASS Collaboration), Proc. Sci. SPIN2018 (2019) 052.

[12] A. V. Efremov, K. Goeke, and P. Schweitzer, Phys. Rev. D 67, 114014 (2003).

[13] A. Bacchetta and M. Radici, Phys. Rev. D 69, 074026 (2004).

[14] S. Gliske, A. Bacchetta, and M. Radici, Phys. Rev. D 90, 114027 (2014); 91, 019902(E) (2015).

[15] A. Bianconi, S. Boffi, R. Jakob, and M. Radici, Phys. Rev. D 62, 034008 (2000).

[16] A. Bacchetta and M. Radici, Phys. Rev. D 67, 094002 (2003).

[17] A. Bacchetta, A. Courtoy, and M. Radici, J. High Energy Phys. 03 (2013) 119.

[18] M. Radici, A. Courtoy, A. Bacchetta, and M. Guagnelli, J. High Energy Phys. 05 (2015) 123.
[19] A. Courtoy, A. Bacchetta, M. Radici, and A. Bianconi, Phys. Rev. D 85, 114023 (2012).

[20] A. Vossen et al. (Belle Collaboration), Phys. Rev. Lett. 107, 072004 (2011).

[21] B. A. Mecking et al., Nucl. Instrum. Methods Phys. Res., Sect. A 503, 513 (2003).

[22] T. Sjostrand, arXiv:hep-ph/9508391.

[23] https://geant4.web.cern.ch/.

[24] B. Pasquini and S. Rodini, Phys. Lett. B 788, 414 (2019).

[25] A. D. Martin, W. J. Stirling, R. S. Thorne, and G. Watt, Eur. Phys. J. C 63, 189 (2009).

[26] R. Jakob, P. J. Mulders, and J. Rodrigues, Nucl. Phys. A626, 937 (1997).

[27] A. I. Signal, Nucl. Phys. B497, 415 (1997); R. L. Jaffe and X. D. Ji, Nucl. Phys. B375, 527 (1992).

[28] Y. Ohnishi and M. Wakamatsu, Phys. Rev. D 69, 114002 (2004); M. Wakamatsu, Phys. Lett. B 509, 59 (2001).

[29] P. Schweitzer, Phys. Rev. D 67, 114010 (2003).

[30] C. Lorcé, B. Pasquini, and P. Schweitzer, J. High Energy Phys. 01 (2015) 103.

[31] A. Courtoy, arXiv:1405.7659.

[32] V. D. Burkert et al. Nucl. Instrum. Methods Phys. Res., Sect. A 959, 163419 (2020).

[33] S. Pisano et al., JLab Experiment E12-06-112B, E12-09008B (2014).

[34] H. Avakian (CLAS Collaboration), Proc. Sci. DIS2019 (2019) 265.

[35] See Supplemental Material at http://link.aps.org/ supplemental/10.1103/PhysRevLett.126.062002 for tables of the kinematic ranges and asymmetry values. 